\title{
Putting noninvasive lung mechanics into context
}

\author{
Peter M.A. Calverley ${ }^{1}$ and Ramon Farre ${ }^{2}$
}

Affiliations: ${ }^{1}$ Institute of Ageing and Chronic Disease, University of Liverpool, Liverpool, UK. ${ }^{2}$ Unitat de Biofisica i Bioenginyeria, Facultat de Medicina, Universitat de Barcelona-IDIBAPS-CIBERES, Barcelona, Spain.

Correspondence: P.M.A. Calverley, Institute of Ageing and Chronic Disease, Faculty of Health and Life Sciences, University of Liverpool, Room 356, 4th Floor, UCD Building, Daulby Street, Liverpool, L69 3GA, UK. E-mail: pmacalaliverpool.ac.uk

0 @ERSpublications

Measuring lung mechanics noninvasively is clinically useful but equipment standardisation is urgently needed http://ow.ly/p9Imj

Anyone who teaches medical students about how the lungs work meets an immediate problem. The best way to describe the mechanics of breathing is in terms of airways resistance and lung compliance but these measurements are not readily available in clinical practice. Instead clinicians focus on the indirect information available from spirometry. The great advantage that spirometry offers is its known immediate reproducibility and limited between test variation in both health and disease [1]. These properties, coupled with its extensive use in clinical decision making, ensure that spirometric measurements are going to be used in managing respiratory disease for the foreseeable future. One particular advantage of spirometry is the availability of age- and height-related normal values against which any recording can be judged [2].

From the 1950s onwards, physiologists realised that applying small multi-frequency pressure oscillations at the mouth would produce changes in volume and flow that provided a direct and noninvasive measurement of respiratory system mechanics. These data were expressed in terms of respiratory system resistance and reactance (the latter a complex concept involving negative numbers that has never appealed to clinicians), and should give an insight into how breathing at rest is related to disease. To make this measurement clinically practical there had to be substantial improvements in the accuracy of the pressure transducers which measured the very small fluctuations in pressure, and hence flow, within the airways and also a considerable increase in the computing power necessary for the complex mathematical manipulations, such as fast Fourier transformations which disentangle the mixture of frequencies applied to describe the behaviour of the respiratory system. By the early 1980s [3] these developments had become a reality and a number of researchers, especially those based in Belgium, made measurements of resistance and reactance in healthy subjects and in several disease states [3-7]. Despite the convenience both in terms of the short duration of measurement and lack of subject cooperation which the technique offered, it was difficult to relate the numbers generated to more familiar measurements made under conditions of forced expiration and so this technique was not widely adopted in adult clinical practice.

The development of commercial systems producing forced oscillation as pseudo-random noise through a loud speaker or as a train of impulses with an impulse shaped pressure oscillation applied every $200 \mathrm{~ms}$ encouraged uptake of the technique in paediatrics [8]. The European Respiratory Society played an important role in producing a document that standardised terminology and methods, which has now been widely adopted [9]. Several groups have used oscillatory methods which average signals over the entire respiratory cycle to evaluate treatment with drugs, especially bronchodilators, in patients with chronic obstructive pulmonary disease (COPD) [10-12], while others have monitored the changes in respiratory system resistance and reactance during recovery from exacerbations from disease $[13,14]$. Work is now on

Received: June 032013 | Accepted after revision: June 102013

Conflict of interest: None declared.

Copyright @ERS 2013 
going to apply home monitoring with these techniques to improve the management of ambulatory patients and to potentially prevent exacerbations $[15,16]$. However, a significant drawback has been the lack of normal data against which results can be assessed, together with concerns about the proposed normal ranges being specific to the equipment on which is was measured.

These deficiencies have now been remedied by the work of OosTVEen et al. [17], as reported in the current issue of the European Respiratory Journal. The authors obtained oscillatory frequency spectra from five different centres, each one using a different machine (two home-made setups and three commercial devices, one of which used the impulse oscillation system), in a group of 368 carefully selected healthy subjects aged 18-80 years. From these data, they were able to compare equipment and developed predicted equations that can be applied generally between patients and systems. Several striking findings emerged from their work.

It is usually accepted that pressure fluctuations at higher frequencies produce lower values for respiratory system resistance and reactance than those at low frequencies, reflecting differences in the degree of transmission of pressure to the periphery to the lungs. Such a pattern was seen with two of the test systems but in two others resistance, but not reactance values, were highest at the high frequencies whilst in the remaining system there was no frequency dependence. This made pooling of data across all the studied frequencies difficult, but also raises questions about the robustness of inferring changes in small airways function from the difference between low and high frequency oscillatory mechanics data. The immediate reproducibility of resistance and reactance data was influenced by the baseline values and was greater than the variation seen with forced expiratory volume in 1 (FEV1), a finding noted in other studies of healthy subjects and COPD patients [18]. Indeed the mean values for impedance variables at $5 \mathrm{~Hz}$ were very similar in this population with a wider age range and sex mix to those reported in an even larger population of older smokers and nonsmokers [18]. The authors studied the effect of a short-acting bronchodilator drug, salbutamol, on respiratory system resistance and reactance. On average, they saw an $11 \%$ fall in resistance values, but based on the presence of a post-bronchodilator value that exceeded the coefficient of reproducibility of the test, almost a third of the healthy subjects were responders. This contrasted with a $17 \%$ response rate seen if FEV1 was used. However, it is important not to confuse the criteria used here with those that have been recommended in American Thoracic Society/European Respiratory Society documents, where an additional minimum volume response is required to define FEV1 reversibility [1]. Moreover, there are issues about the impact of baseline lung function and whether the absolute volume change in a disease like COPD is really different after use of a bronchodilator from that seen in healthy subjects [19]. The current data suggest that bronchodilators affect airways smooth muscle tone in healthy people and this may provide a sensitive way of exploring the impact of new therapies, which are intended to act primarily as bronchodilators.

Inevitably, there are some limitations. The data from one machine showed much more frequency dependence for the impedance values than the others. Whether this reflects differences in the circuit used, the device selected, the individuals studied or the way in which oscillations were delivered should be resolved. All the data were recorded without reference to the end-expiratory lung volume (EELV) at which the patients were breathing. This is an important variable to record as small differences in EELV impact on the measured resistance and reactance. Moreover, the results from the study of OOSTVEEN et al. [17] are presented as the average of data collected over the whole respiratory cycle. This is acceptable in healthy subjects, but raises problems when air flow obstruction develops as resistance, and particularly reactance, values during expiration become significantly greater than those during inspiration, particularly when expiratory flow limitation is present [20]. This can be important in disease states where the measurement of inspiratory reactance is closely related to transpulmonary resistance [21], can distinguish patients with asthma from those with COPD [22] and appears to be a better guide to the effectiveness of inhaled bronchodilator treatment [23]. Future normal values developed in a manner similar to those collected here should report data partitioned according to the phase of the respiratory cycle in which it is collected.

OOSTVEEN et al. [17] used an analogue reference system with an impedance high enough to simulate that of a patient and took care to define the technical requirements of the hardware used (e.g. range, linearity, frequency response, common-mode rejection ratio, and sensitivity to vibrations) as previously described [9]. However, there is still a need to standardise the performance of the software so that it is capable of reproducing standard wave forms accurately as is done when validating new spirometers [2]. Developing a system to accurately produce such complex waveforms superimposed on tidal breathing would be a complex task. However, a simplified version using a set of pressure and flow digital signals simulating measurements made in patients presenting with a range of pre-defined impedance values and including the signal components corresponding to different real patterns of spontaneous breathing could be produced relatively easily. There is a need for an expert group to develop such a device and distribute it as a "gold standard" to those who produce oscillatory systems. 
The data from OostveEn et al. [17] represents an important step forward in the field of oscillatory mechanics. We can now identify when data are abnormal relative to values from healthy subjects, over a wide range of ages and applicable to both sexes. These results appear more robust than those in earlier data sets, but can undoubtedly be improved by other workers adding additional information. The greater challenge for clinical physiologists is no longer in the interpretation of whether their data are "normal or abnormal" but in deciding the clinical significance of these measurements made during tidal breathing. The interpretation of oscillatory mechanics is not simple, but its application to clinical medicine opens up exciting possibilities for better patient monitoring and increased understanding of the impact of disease on the individual.

\section{References}

1 Pellegrino R, Viegi G, Brusasco V, et al. Interpretative strategies for lung function tests. Eur Respir J 2005; 26 : 948-968.

Miller MR, Hankinson J, Brusasco V, et al. Standardisation of spirometry. Eur Respir J 2005; 26: 319-338.

3 Pasker HG, Schepers R, Clement J, et al. Total respiratory impedance measured by means of the forced oscillation technique in subjects with and without respiratory complaints. Eur Respir J 1996; 9: 131-139.

4 Farre R, Rotger M, Marchal F, et al. Assessment of bronchial reactivity by forced oscillation admittance avoids the upper airway artefact. Eur Respir J 1999; 13: 761-766.

5 Farre R, Peslin R, Rotger M, et al. Forced oscillation total respiratory resistance and spontaneous breathing lung resistance in COPD patients. Eur Respir J 1999; 14: 172-178.

6 Van Noord JA, Clement J, Van de Woestijne KP, et al. Total respiratory resistance and reactance in patients with asthma, chronic bronchitis, and emphysema. Am Rev Respir Dis 1991; 143: 922-927.

7 Clement J, Landser FJ, Van de Woestijne KP. Total resistance and reactance in patients with respiratory complaints with and without airways obstruction. Chest 1983; 83: 215-220.

8 Beydon N, Davis SD, Lombardi E, et al. An official American Thoracic Society/European Respiratory Society statement: pulmonary function testing in preschool children. Am J Respir Crit Care Med 2007; 175: 1304-1345.

9 Oostveen E, MacLeod D, Lorino $\mathrm{H}$, et al. The forced oscillation technique in clinical practice: methodology, recommendations and future developments. Eur Respir J 2003; 22: 1026-1041.

10 Crim C, Celli B, Edwards LD, et al. Respiratory system impedance with impulse oscillometry in healthy and COPD subjects: ECLIPSE baseline results. Respir Med 2011; 105: 1069-1078.

11 Borrill ZL, Houghton CM, Tal-Singer R, et al. The use of plethysmography and oscillometry to compare longacting bronchodilators in patients with COPD. Br J Clin Pharmacol 2008; 65: 244-252.

12 Kolsum U, Borrill Z, Roy K, et al. Impulse oscillometry in COPD: identification of measurements related to airway obstruction, airway conductance and lung volumes. Respir Med 2009; 103: 136-143.

13 Stevenson NJ, Walker PP, Costello RW, et al. Lung mechanics and dyspnea during exacerbations of chronic obstructive pulmonary disease. Am J Respir Crit Care Med 2005; 172: 1510-1516.

14 Johnson MK, Birch M, Carter R, et al. Measurement of physiological recovery from exacerbation of chronic obstructive pulmonary disease using within-breath forced oscillometry. Thorax 2007; 62: 299-306.

15 Donaldson GC, Seemungal TA, Hurst JR, et al. Detrended fluctuation analysis of peak expiratory flow and exacerbation frequency in COPD. Eur Respir J 2012; 40: 1123-1129.

16 Gonem S, Umar I, Burke D, et al. Airway impedance entropy and exacerbations in severe asthma. Eur Respir J 2012; 40: 1156-1163.

17 Oostveen E, Boda K, van der Grinten CPM, et al. Respiratory impedance in healthy subjects: baseline values and bronchodilator response. Eur Respir J 2013; 42: 1513-1523.

18 Crim C, Celli B, Edwards LD, et al. Respiratory system impedance with impulse oscillometry in healthy and COPD subjects: ECLIPSE baseline results. Respir Med 2011; 105: 1069-1078.

19 Albert P, Agusti A, Edwards L, et al. Bronchodilator responsiveness as a phenotypic characteristic of established chronic obstructive pulmonary disease. Thorax 2012; 67: 701-708.

20 Dellaca RL, Duffy N, Pompilio PP, et al. Expiratory flow limitation detected by forced oscillation and negative expiratory pressure. Eur Respir J 2007; 29: 363-374.

21 Johnson MK, Birch M, Carter R, et al. Use of reactance to estimate transpulmonary resistance. Eur Respir J 2005; 25: 1061-1069.

22 Paredi P, Goldman M, Alamen A, et al. Comparison of inspiratory and expiratory resistance and reactance in patients with asthma and chronic obstructive pulmonary disease. Thorax 2010; 65: 263-267.

23 Dellaca RL, Pompilio PP, Walker PP, et al. Effect of bronchodilation on expiratory flow limitation and resting lung mechanics in COPD. Eur Respir J 2009; 33: 1329-1337. 